\title{
Industrial CryoEM: reasons why and examples of impact
}

\author{
C Phillips ${ }^{1}$ \\ ${ }^{1}$ AstraZeneca, Cambridge, UK \\ chris.phillips@astrazeneca.com
}

This talk will focus on the reasons why Pharmaceutical Companies such as AstraZeneca are investing in building cryoEM capabilities and give some early examples of impact in drug discovery projects, not only through traditional structure based design approaches but also through insights into the molecular basis of disease process. Specifically the talk will describe the complex of phospholipase C gamma with the FGFR1 kinase domain and insights into the mechanism of disease relevant mutations.

Acta Cryst. (2020). A76, a162 